\title{
La organización del estudio instrumental: un factor incidente en las molestias músculo esqueléticas en clarinetistas*
}

THE PRACTICE HABITS AND SCHEDULES OF MUSICIANS: AN INCIDENTAL FACTOR IN THE SKELETAL MUSCLE DISCOMFORT FOR CLARINET PLAYERS

A ORGANIZAÇÃO DO ESTUDO DO INSTRUMENTO: UM FATOR INCIDENTE NO MÚSCULO ESOUELÉTICO EM CLARINETISTAS

\section{Andrea Patricia Calvo Soto** Edilberto Castaño Betancourt*** Esperanza Gómez Ramírez****}

Cuadernos de Música, Artes Visuales y Artes Escénicas

/ Volumen 11 - Número 1 / enero - junio de 2016

/ ISSN 1794-6670/ Bogotá, D.C., Colombia / pp. 55-65

Fecha de recepción: 26 de septiembre de 2015 | Fecha de aceptación: 26 de octubre de 2015 | Disponible en línea: 30 de mayo de 2016. Encuentre este artículo en http://cuadernosmusicayartes.javeriana.edu.co/ doi: 10.11144/Javeriana.mavae11-1.oeif

* Artículo de Investigación

** Fisioterapeuta, Magíster en Salud Ocupacional. Docente Fisioterapia Fundación Universitaria María Cano. Extensión Cali, Colombia.

** Licenciado en Música. Docente en el Programa de Extensión Universidad del Valle CaliColombia y en la Escuela de Música Fundación SIDOC.

**** Fisioterapeuta, Magíster en Educación. Docente de Fisioterapia en la Fundación Universitaria María Cano. Extensión Cali, Colombia. 


\section{Resumen}

Se realizó una investigación para conocer los hábitos de estudio de estudiantes de clarinete, en tres instituciones de la ciudad de Cali, Colombia: un estudio descriptivo transversal, que usó una encuesta sobre los tiempos de estudio, posturas, periodos de descanso, entre otros. La muestra se realizó con 12 estudiantes entre 18 y 36 años. Ellos respondieron que llevaban más de cinco años de interpretación del instrumento y estudiaban entre tres y seis días a la semana, de dos a tres sesiones diarias. Practicaban entre una hora y media y tres horas. Estudiaban sin pausa por 40 minutos o más, las pausas fueron variables. No realizaban ejercicio físico o de calentamiento/entrenamiento. La prueba de Fisher $p=0,061$ mostró una tendencia alta en relación con la duración de sesiones de estudio regular y de estudio para recitales, exámenes o presentaciones. No hubo diferencias significativas entre los hábitos de estudio y el nivel de formación. El estudio permite concluir que los estudiantes participantes de la muestra no tienen una forma estandarizada de estudio.

Palabras clave: músicos; estudio musical; clarinetistas

\section{Abstract}

A research was carried out to know the study habits of students of clarinet, in 3 institutions of the city of Cali, Colombia: A descriptive cross-sectional study, using a survey of the study time, posture, rest periods, etc. The sample was 12 students between 18 and 36 years. Responded that took more than 5 years of interpretation and studying 3 to 6 days a week, 2 to 3 sessions per day. Practiced between an hour and a half and 3 hours. Studying without pause for 40 minutes or more, the breaks were variables. They did not exercise or heat/training. Fisher's exact test $p=0,061$ showed a tendency toward high in relation to the duration of regular study sessions and study for recitals, exams or presentations. There were no significant differences between the study habits and the level of training. Conclusion: students do not have a standardized way to study.

Keywords: music; music education; clarinetist

\section{Resumo}

Foi realizado um levantamento para conhecer os hábitos de estudo dos alunos de clarinete, em 3 instituições da cidade de Cali, Colômbia. Um estudo descritivo de corte transversal, com um levantamento dos períodos de estudo, postura, períodos de repouso etc. A amostra foi de 12 alunos entre 18 e 36 anos. Responderam que levou mais de 5 anos de interpretação e estudo 3 a 6 dias por semana, 2 a 3 sessões por dia. Praticada entre uma hora e meia a 3 horas. Estudando sem parar por 40 minutos ou mais, as quebras foram variáveis. Não exercício ou calor/formação. O teste exato de Fisher $p=0,061$ mostrou uma tendência a alta em relação à duração das sessões de estudo regular e estudar para os considerandos, exames ou apresentações. Não foram observadas diferenças significativas entre os hábitos de estudo e o nível de formação. Conclusão: os alunos não têm uma forma padronizada de estudo.

Palavras-chave: músicos; estúdio musical; clarinetistas 


\section{INTRODUCCIÓN}

Cuando se está frente a un instrumentista profesional se disfruta de su ejecución, pero poco se indaga sobre cuáles destrezas tuvo que desarrollar para alcanzar ese grado de maestría. Se reconoce que para tocar cualquier instrumento es importante perfeccionar varias cualidades que se van dominando con el tiempo. El músico, para llevar a cabo sus tareas interpretativas, debe emplear muchas veces movimientos repetitivos, fuerza, precisión, coordinación, a veces posturas y ángulos articulares poco naturales o fisiológicos (Roset-Llobet, RosinésCubells y Saló-Orfila, 2000, p. 172). Algunos autores señalan que el manejo de las jornadas de estudio en los músicos influye en la presentación de síntomas de dolor músculo esquelético, incluso cuando se está en proceso de formación, lo cual indica que no es un problema exclusivo de los músicos profesionales.

Si se consideran todas las exigencias físicas corporales de un músico, se entienden las razones por las cuales son víctimas de dolor y problemas músculo esqueléticos en el transcurso de sus carreras. Estos problemas se deben a la interacción de múltiples factores individuales, pero también existen situaciones propias de cada instrumento que favorecen la aparición de síndromes por sobreuso (Hansen y Reed, 2006, p. 720). Entre los problemas asociados están: el desarrollo de la técnica de interpretación, tocar por tiempo prolongado, así como las características físicas que tiene el instrumento, la exigencia del repertorio y el grado de entrenamiento del músico (Frank y Von Mühlen, 2007, p. 189).

Autores como Viaño (2010, p. 84) señalan otros aspectos contribuyentes como: los tiempos de ensayo e interpretación y la forma de organización de estos, además de otras variables, entre las que se cuentan: el volumen de ensayo, la frecuencia, duración e intensidad de las sesiones de estudio; descansos o recuperaciones durante las sesiones, el número de sesiones al día, a la semana, y el horario habitual de práctica.

En consideración a esta situación, los investigadores se interesaron en indagar acerca de los hábitos de estudio en estudiantes universitarios de instrumento en la ciudad, encontrándose en un primer sondeo que fueron los clarinetistas quienes más reportaron a sus maestros sintomatología músculo esquelética, debida a su práctica instrumental. Por esta razón, se profundizó en cómo eran los hábitos de estudio y la organización de las sesiones de práctica de los alumnos de clarinete.

\section{METODOLOGÍA}

El presente trabajo está enmarcado en el enfoque cuantitativo, es un estudio descriptivo de corte transversal.

\section{Muestra}

Se contó con una muestra de doce estudiantes de clarinete en pregrado, de la ciudad de Cali, Colombia, se llevó a cabo un muestreo por conveniencia, en el que se seleccionó a las personas que accedieran a participar del estudio, diligenciaran el consentimiento informado y estuvieran matriculados en cualquier nivel de la asignatura de instrumento. Hicieron parte de 
la investigación estudiantes de las tres instituciones universitarias de la ciudad que ofrecen programas académicos de música. La población general fue de 18 estudiantes de clarinete en estas tres universidades.

\section{Procedimientos}

Se aplicó un cuestionario que fue construido por los investigadores, se validó a través de la evaluación de un panel de expertos: tres músicos profesionales quienes comentaron acerca de la comprensión de las preguntas y posibles ambigüedades en las mismas. Posteriormente el cuestionario fue revisado por un estadístico epidemiólogo, quien emitió juicio sobre su construcción y se hicieron cambios de acuerdo a sus sugerencias. A continuación, se aplicó una prueba piloto a quince estudiantes de instrumento (diferentes al clarinete) con características similares a la población que se iba a estudiar, en una de las instituciones de educación superior. Esta prueba tenía el objetivo de contrastar si la encuesta funcionaba como se pretendía, si se adecuaba a los objetivos de la investigación y para detectar posibles errores. ${ }^{1}$

La encuesta de hábitos de estudio constaba de 20 preguntas, entre abiertas y cerradas, sobre: presencia de síntomas músculo esqueléticos en algún momento de la práctica, nivel de formación, tiempos de estudio regular y para recital (diario y semanal), horario habitual de ensayo, tiempo de pausas durante las sesiones, posturas de estudio, la duración de las sesiones individuales y el número de periodos de descanso en una sesión, uso de accesorios, presencia de rutina de entrenamiento físico y realización de ejercicio regular. Este cuestionario se aplicó a manera de entrevista individual y presencial.

Entre los métodos estadísticos que se aplicaron están el análisis descriptivo, también pruebas de asociación utilizando chi cuadrado y prueba de Fisher con un nivel de significancia estadística de $p<0,05$.

\section{RESULTADOS}

Se distribuyó la muestra en siete hombres y cinco mujeres, con edades entre 18 y 36 años y una moda de 19 años. Con respecto al nivel de formación, la mayoría estaba en el nivel intermedio y avanzado ( $n=8)$, lo que constituye estar en la educación formal por más de dos años (Tabla 1). De los doce alumnos estudiados, siete de ellos llevaban más de cinco años en la interpretación de este instrumento de viento (Gráfica 1).

Tabla 1. Nivel de formación de los estudiantes de clarinete $(n=12)$

\begin{tabular}{lc}
\hline Nivel de formación & Frecuencia \\
\hline Básico & 4 \\
Intermedio & 3 \\
\hline Avanzado & 5 \\
\hline Total & $\mathbf{1 2}$ \\
\hline
\end{tabular}

FUENTE: Elaboración propia 


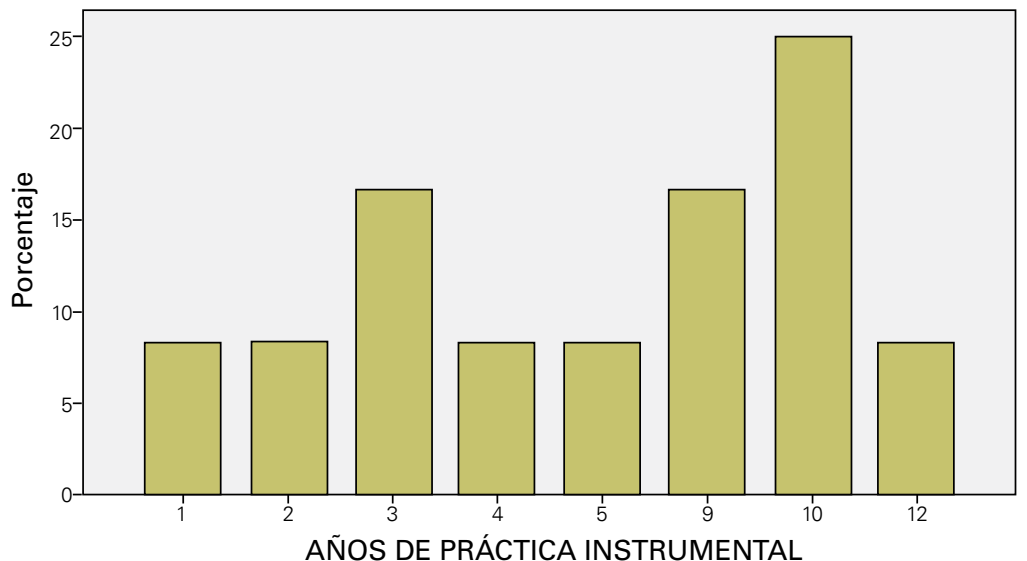

Gráfica 1. Distribución de los años de práctica instrumental de los estudiantes de clarinete $(n=12)$ FUENTE: Elaboración propia

El total de estudiantes de la muestra $(n=12)$ reportó presentar en algún momento molestias músculo esqueléticas relacionadas con la práctica del clarinete durante su formación, en el brazo-antebrazo, pulgar o alrededor de la boca. Solo uno de ellos manifestó síntomas lo suficientemente complejos como para requerir manejo y un periodo de paro de las actividades.

En relación con la jornada de estudio, la mayoría estudiaba entre tres y seis días a la semana y no practicaban a una sola jornada diaria, sino que distribuían su tiempo en dos o tres sesiones diarias (Gráfica 2), siendo en total discontinua: en la mañana-tarde, la tarde-noche y mañana-tarde-noche (Tabla 2).

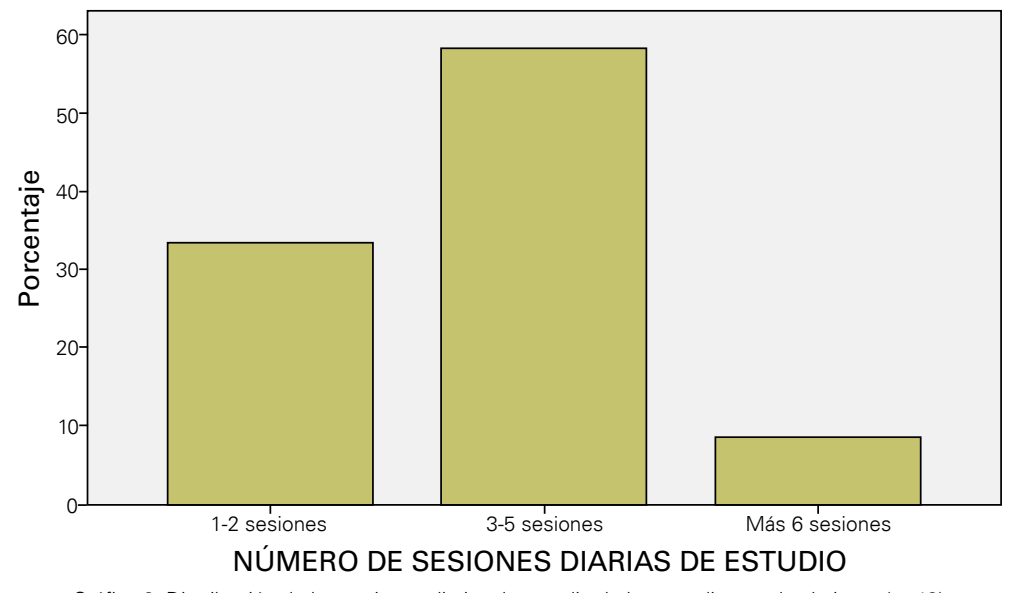

Gráfica 2. Distribución de las sesiones diarias de estudio de los estudiantes de clarinete $(n=12)$ FUENTE: Elaboración propia

Tabla 2. Frecuencia de las jornadas diarias de estudio de clarinete $(n=12)$

\begin{tabular}{lc}
\hline Jornadas de estudio diarias & Frecuencia \\
\hline Mañana & 1 \\
Noche & 1 \\
Mañana/tarde & 4 \\
\hline Tarde/noche & 1 \\
Mañana/noche & 2 \\
Mañana/tarde/noche & 3 \\
\hline Total & $\mathbf{1 2}$ \\
\hline
\end{tabular}

FUENTE: Elaboración propia 
Tres cuartas partes del grupo de estudio tenían en la jornada más de una hora y media de práctica, y en algunos podía llegar hasta tres horas (Tabla 3). En ese tiempo la mayoría estudiaba sin pausa por 40 minutos, o incluso por más tiempo (Gráfica 3).

Tabla 3. Frecuencia de la duración de las sesiones de estudio del clarinete $(n=12)$

\begin{tabular}{lc}
\hline Duración de la sesión completa de estudio & Frecuencia \\
\hline 30-60 minutos & 2 \\
61-91 minutos & 1 \\
\hline $92-122$ minutos & 3 \\
\hline 123-153 minutos & 3 \\
\hline Más 154 minutos & 3 \\
\hline Total & $\mathbf{1 2}$ \\
\hline
\end{tabular}

FUENTE: Elaboración propia

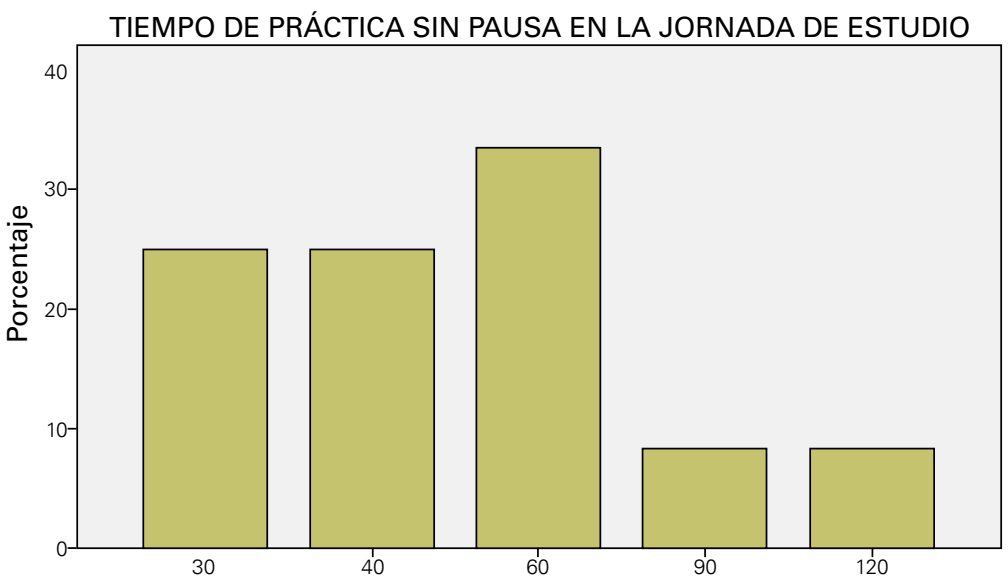

Gráfica 3. Distribución del tiempo de práctica instrumental sin pausa de los estudiantes de clarinete $(n=12)$ FUENTE: Elaboración propia

La mitad de los alumnos realizaban entre dos y tres pausas, descansaban después de 40 minutos a una hora de estar interpretando el clarinete (Gráfica 4). La duración de las pausas fue entre cinco y nueve minutos para la mayoría, aunque hay alumnos que no hacían pausas, o las hacían menos de cinco minutos o muy largas, de más de quince minutos.

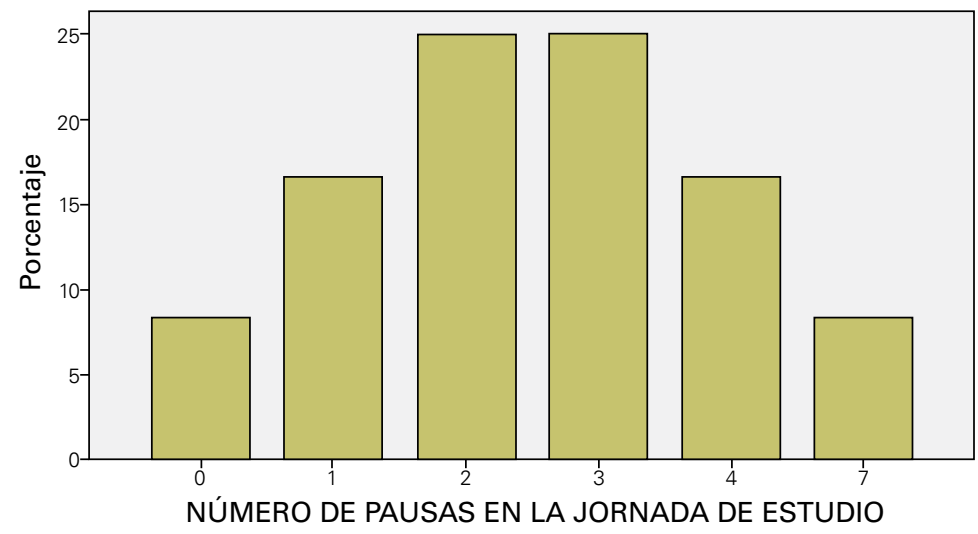

Gráfica 4. Distribución del número las pausas durante la jornada de estudio del clarinete $(n=12)$ FUENTE: Elaboración propia 
La mayoría de los estudiantes (9 de los 12 alumnos) practicaba en posición sentada. Se identificó que solo uno de los alumnos usaba accesorio para soportar el clarinete. (Gráfica № 5 ).

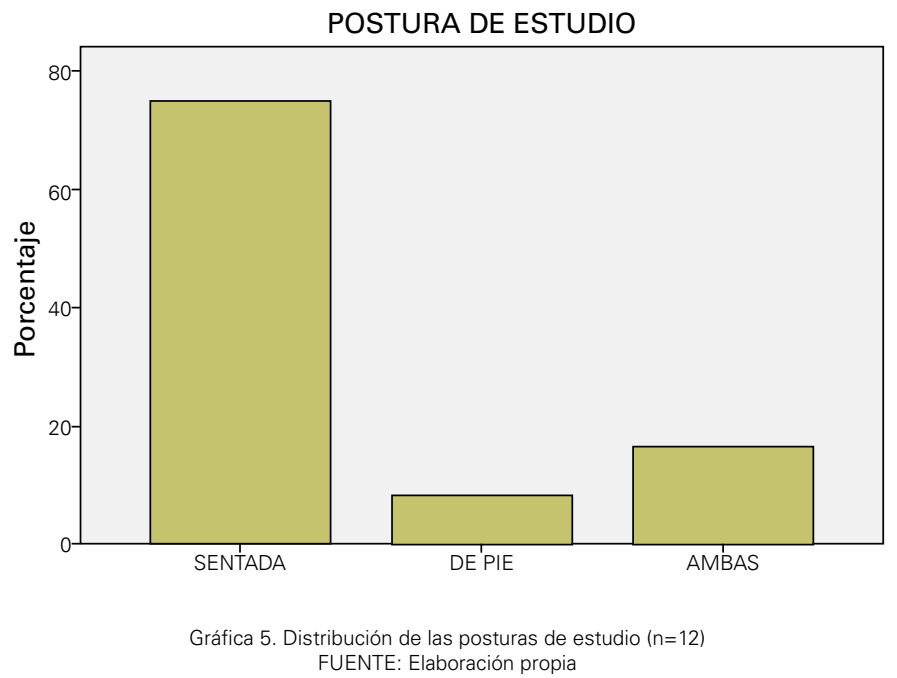

Con respecto a la preparación física, más de la mitad de los alumnos respondieron que no realizaban ejercicios de calentamiento, ejercicios preparatorios para ejecutar el instrumento. Aunque algunos realizaban deporte $(n=5)$, la frecuencia del mismo en la semana es muy baja, reportan dos veces a la semana u ocasionalmente, hablando en términos de una vez por mes, o más espaciado a una vez cada dos meses; en su orden los deportes más comunes fueron: ciclismo, natación y fútbol.

Las horas de estudio reportadas se incrementaron casi al doble cuando se preparaban para un recital o presentaciones, siendo más relevante para aquellos que estaban en semestres avanzados, porque tienen una práctica más frecuente debido a la participación en grupos o conjuntos externos. En cuanto a esto, la frecuencia de presentaciones, recitales o exámenes, varió entre una y cinco veces en el semestre académico (cuatro meses).

Con respecto al tiempo de estudio durante la práctica regular y la práctica para exámenes y presentaciones se utilizaron las pruebas de Fisher, que arrojaron un valor $p=0,061$, mostrándose una tendencia alta en relación con la duración de sesiones de estudio regular y de estudio para recitales, exámenes o presentaciones.

No hubo diferencias estadísticamente significativas entre los hábitos de estudio (tiempos de estudio, jornadas, etcétera) y el nivel de formación.

\section{Discusión}

Viaño (2009, p. 13) estableció con relación a los síntomas músculo esqueléticos ocasionados por la práctica musical, que los intérpretes empiezan a tocar mucho antes de que se haya finalizado el crecimiento de su sistema músculo esquelético. Ello fue corroborado en esta población de estudiantes universitarios, pues con relación a la moda en edad de 19 años, varios de ellos empezaron a practicar el clarinete antes de los quince años. Con ello se refuerza la tesis de Spahn, Nusseck y Zander (2014, p. 8), quien indica que los problemas de salud que 
experimentan los músicos no inician con la vida profesional sino durante etapas tempranas de su educación musical, puede ser en la infancia y especialmente en el entrenamiento universitario.

Con respecto al tiempo de formación, más de la mitad de los estudiantes de la presente investigación están por encima de los cinco años de estudio del clarinete; sin embargo este dato se ubica por debajo del estudio de Arnason, Arnason y Briem (2014, p. 75), cuyos resultados en estudiantes de música con desórdenes músculo esqueléticos en Islandia, fue de 11,2 años para el instrumento principal.

Todos los estudiantes de la muestra reportaron molestias músculo esqueléticas en algún momento de la formación, valor superior al que presenta el trabajo de Sphan, en donde el $51 \%$ de los estudiantes referenciaron tener problemas relacionados con la interpretación, mínimo una vez durante su vida de estudio (2014, p. 13); otros estudios llegan a reportar mediciones de hasta el $81 \%$ de experiencia de dolor y disconfort durante la interpretación del instrumento (Steinmetz, 2012, p. 628).

Las molestias mencionadas se presentaron principalmente en el brazo, pulgar o alrededor de la boca, lo cual se relaciona con lo establecido por Fry:

El sobreuso de los clarinetistas típicamente afectó primeramente y de forma más profunda los músculos del primer espacio interóseo de la mano derecha y los ligamentos de la base del pulgar y del lado radial de la muñeca, lo anterior por la contracción muscular sostenida. (1987, p. 38)

Este mismo aspecto contrasta en músicos profesionales de viento madera, donde el OR de los síntomas músculo esqueléticos puntuó más para los siguientes segmentos corporales: espalda alta, codo derecho, mano y muñeca derecha. Pese a ese hallazgo, fueron estos interpretes dentro de la orquesta sinfónica los que tuvieron el riesgo más bajo de síntomas músculo esqueléticos, y el menor riesgo de impacto sobre la función (Paarup et al., 2011, p. 10).

En los estudiantes objeto de esta investigación, tres cuartas partes del grupo tenía una jornada de más de una hora y media de estudio, y en algunos podía llegar hasta tres horas. La mayoría estudiaba entre tres y seis días a la semana y no practicaba a una sola jornada diaria, sino que distribuían su tiempo en dos o tres sesiones diarias. Estos valores son inferiores a los presentados en el trabajo de Arnason, Arnason y Briem, pues los estudiantes de su investigación practicaban en promedio 6,5 horas semanales, en promedio de seis días a la semana, dos veces por día (2014, p. 75).

Con respecto a las pausas durante el tiempo de práctica, la mitad de los alumnos realizan entre dos y tres pausas y descansan después de 40 minutos a una hora de estar interpretando el clarinete. Para los autores mencionados la sesión duraba 74 minutos, con un mínimo de descanso de 39 minutos (Arnason, Arnason y Briem, 2014, p. 75), en el trabajo de Steinmetz el $18,2 \%$ practicó sin una pausa de al menos diez minutos y otro $18,2 \%$ con una pausa mayor de diez minutos (2012, pp. 628-629). Fue evidente en el grupo de alumnos de esta investigación que no tienen estandarizado cada cuánto tiempo deben hacer una pausa y cuánto tiempo debe durar esa pausa, debido a que muchos de ellos la toman cuando ya tienen sensación de cansancio. La variabilidad en los tiempos de pausa muestra que no hay claridad en cuándo se debe descansar, después de haber realizado una actividad tan exigente como la interpretación instrumental. Según Velásquez (2005, p. 129), dentro de los conceptos ergonómicos de recuperación, se deben considerar pausas cortas y frecuentes, que son más efectivas que las 
pausas largas y espaciadas. Por lo tanto, en el caso de los estudiantes de clarinete hay falta de información sobre cómo debe administrarse la sesión de estudio instrumental.

En el estudio de Prevalence of sobreuse (injury) syndrome in australian music schools, se encontró una correlación del 100\% entre el inicio de los síntomas y el aumento del tiempo de estudio, además de una intensidad multiplicada cuando los alumnos se prepararon para un examen, un recital, una competición y la práctica de un nuevo estudio (Fry, 1987, p. 38). Este trabajo de investigación corroboró que durante la preparación de un recital o de una presentación, los estudiantes aumentan el tiempo de práctica, y con ello hubo también un incremento en la exposición a presentar síntomas de dolor.

Dentro de los factores de riesgo de problemas músculo esqueléticos en músicos, la literatura describe que el promedio de horas de estudio diarias, los tiempos de interpretación prolongados, inadecuados tiempos de descanso, y para este caso prepararse para una presentación, influyen en el desarrollo de estas disfunciones (Kaufman-Cohen y Ratzol, 2011, p. 93). Por tanto, al hablar de los aspectos de tipo organizacional de la práctica musical que están relacionados con los desórdenes músculo esqueléticos, se menciona que debe hacerse un adecuado manejo de las sesiones de estudio, pues las largas jornadas o en particular la intensidad de la práctica, se constituye en el más importante determinador de dolor, incluso más que el total de horas gastadas en la práctica (Fry y Rowley, 1989, p. 1001).

El tiempo de duración de la sesión juega un papel crucial, para ello se debe establecer un periodo de estudio por día y organizarlo por ciclos, de tal manera que se consideren periodos de ejecución donde va a haber tensión para algún segmento corporal, y también tiempo de pausa para la recuperación. De ninguna manera se trata de tocar a pesar del dolor; autores como Fry (1987, p. 39) ya habían señalado una sugerencia al respecto, descansar aproximadamente por cinco minutos por cada 25 minutos de práctica instrumental.

En cuanto a la realización de actividades complementarias de entrenamiento y ejercicio, más de la mitad de los alumnos respondieron que no realizaban ejercicios de calentamiento o ejercicios preparatorios para ejecutar el instrumento, y que aunque hacían deporte $(n=5)$, la frecuencia del mismo en la semana era muy bajo, con reportes de dos veces a la semana o hasta cada mes o dos meses (ciclismo, natación y fútbol). En otros trabajos los estudiantes presentaron mayores medidas preventivas, pues el 91\% de los sujetos de estudio hacían deportes y un $42 \%$ utilizaba técnicas de relajación como las técnicas Feldenkrais, Alexander o dispokinesis (Spahn, Nusseck y Zander, 2014, p. 11). Implementar estas actividades y tener programas en las instituciones de formación permite que los estudiantes tomen conciencia de los beneficios de promover la salud en su vida diaria.

\section{CONCLUSIONES}

Para finalizar, se comenta que en lo que respecta a la preparación para la acción, el intérprete requiere convertirse en casi "un atleta de miembro superior", debido al nivel y tipo de habilidades motrices requeridas (Elbaum, 1986, p. 286). En esta investigación se evidenció que los estudiantes no hacen sesiones de entrenamiento físico o de ejercicio regular, por tanto no perciben la importancia de la preparación física para la ejecución instrumental. Pese a este hallazgo, se considera que debe tenerse en cuenta la condición física y su entrenamiento, para responder a las demandas físicas de la interpretación. En instrumentistas de viento, por ejemplo, se han relacionado ciertas cualidades físicas con el tocar un instrumento, como serían la 
coordinación, flexibilidad, velocidad, potencia y resistencia. Se requiere velocidad para permitir un allegro, solo la apropiada potencia produce un fortissimo, sin la demanda de resistencia de una ópera de cuatro horas esta no puede ser sostenida (Hahnengress, 2003, p.149). Los intérpretes, por lo tanto, deben tener una rutina de ejercicio y un entrenamiento de las cualidades físicas para adaptar el cuerpo al trabajo, y de modo que frente a una situación de exigencia se pueda responder de la mejor forma, evitando la aparición de fatiga y lesiones.

Cuando el músico conoce las tareas preparatorias del trabajo, e interviene sobre las variables de exigencia de fuerza muscular, de postura y de tiempo, comprende que el dominio del instrumento no se alcanza solo tocándolo, sino que se debe perfeccionar mediante la práctica de tres actividades: trabajo con la expresión individual y musical, trabajo con el instrumento y trabajo sin el instrumento (Klein-Volgelbach, 2010, p.118). Respecto a otras variables adicionales, se encuentran factores medioambientales específicos (ruido, iluminación y ventilación), características del espacio, exposición a la vibración, tener que cumplir las exigencias de calidad en la interpretación y el montaje del repertorio. De esa forma se considera importante atender a las características de los elementos que el músico usa, que incluyen el mobiliario - las sillas, los soportes y accesorios- así como algunos recursos ergonómicos como apoyos, almohadillas y cordeles entre otros, que van a influir directamente en las posturas que se adoptan durante el estudio del instrumento.

Estos son temas que deben ser abordados en el aula de clase, y ser considerados como parte de las temáticas planteadas para el perfeccionamiento de la técnica de interpretación. Maestros y estudiantes deben conocer aspectos generales de ergonomía para músicos, con el fin de tocar con maestría sin correr el riesgo de presentar lesiones músculo esqueléticas.

\section{NOTAS}

1 El instrumento musical no fue sometido a validación dado que como investigadores se consideró que el ejercicio realizado de verificación con el panel de expertos, colegas pares y estudiantes ofrece un nivel de confiabilidad suficiente para los alcances del proyecto.

\section{REFERENCIAS}

Arnason, Kristján, Arnason, Arni y Briem, Kristin. "Playing-related musculoskeletal disorders among icelandic music students: differences between students playing classical vs rhythmic music". Med Probl Perform Art, Englewood, vol. 29, núm. 2, 2014: 74-9.

Elbaum, Leonard. "Musculoskeletal problems of instrumental musicians". Journal of orthopedic and sport physical therapy, Alexandria, vol. 6, 1986: 285-287.

Frank, Annemarie y Von Mühlen, Carlos Alberto. “Queixas Musculoesqueléticas em Músicos: prevalência e fatores de risco". Brazilian Journal of Rheumatology, São Paulo, vol. 47, núm. 3, 2007: 188-196.

Fry, Henry Jacob. "Occupational Maladies of Musicians: Their Cause and Prevention". International Journal of Music Education, Malvern, vol. 4, núm. 1, 1984: 59-63.

Fry, Henry Jacob. "Prevalence of overuse (injury) syndrome in Australian music". British Journal of Industrial Medicine, London, vol. 44, 1987: 35-40. 
Fry, Henry Jacob y Rowley, Ann. "Music related upper limb pain in schoolchildren". Rheum Dis. London, vol. 48, núm. 12, 1989: 998-1002.

Hahnengress, Maria. "Auf dem Weg zur Leistungs und Aufführungsphysiologie. Teil II: Leistungsphysiologische Prinzipien", Musikphysiologie und Musikermedizin, vol. 10, núm. 3, Berlin, 2003: 149-161.

Hansen, Pamela y Reed, Kristi. "Common Musculoskeletal Problems in the Performing Artist". Physical Medicine Rehabilitation Clinics of North America, Philadelphia, vol. 16, 2006: 789-801.

Roset-Llobet, Jaume, Rosinés-Cubells, Dolors y Saló-Orfila, Josep M. “Identification of Risk Factors for Musicians in Catalonia (Spain)". Medical Problems of Performing Artists, Englewood, vol. 15, 2000: 167-174.

Kaufman-Cohen, Yael y Ratzon, Navah. "Correlation between risk factors and musculoskeletal disorders among classical musicians". Occupational Medicine, Philadelphia, vol. 61, núm. 2, 2011: 90-95.

Klein-Volgelbach, Suzzanne. Interpretación musical y postura corporal. Madrid: Akal, 2010.

Luftmann, Alwin y otros. La prévention des troubles musculo-squelettiques sur le lieu de travail. Paris: Organisation Mondial de la Santé. Série Protection de la santé des travailleurs $N^{\circ} 5$. 2004.

Paarup, Helene, Baelum, Jesper, Holm, Jonas, Manniche, Claus y Wedderkopp, Niels. “Prevalence and consequences of musculoskeletal symptoms in symphony orchestra musicians vary by gender: a cross-sectional study". BMC Musculoskelet Disord. London, vol.7, 2011: 212-223.

Spahn, Claudia, Nusseck, Manfred y Zander, Mark. "Long-term analysis of health status and preventive behavior in music students across an entire university program". Med Prob/ Perform Art, Englewood, vol. 29, núm. 1, 2014: 8-15.

Steinmetz, Anke, Möller, Heiko, Seidel, Wolfram y Rigotti, Thomas. "Playing-related musculoskeletal disorders in music students-associated musculoskeletal signs ". Eur J Phys Rehabil Med, Torino, vol. 48, 2012: 625-633.

Velásquez, Juan Carlos. Carga física de trabajo. Bases fisiológicas y metodológicas para su estudio. Pereira: Universidad Libre, 2005: 128-130.

Viaño, Santasmarinas, Jorge. Actividad física, actividad musical y trastornos músculo-esqueléticos en estudiantes instrumentistas de conservatorio. Tesis Doctoral. Universidad de Vigo, Facultad de Ciencias de la Educación y del Deporte, Departamento de Didácticas Especiales, 2009. [En línea] http://issuu.com/jorgeviano/docs/tesis_doctoral_jorge_viano (Acceso: 13 marzo de 2015).

Viaño Santasmarinas, Jorge et al. "Trastornos músculo-esqueléticos (TMRIs) en músicos instrumentalistas estudiantes de secundaria y universitarios". Revista de Investigación en Educación, Vigo, vol.

8, 2010: 83-96.

\section{Cómo citar este artículo:}

Calvo, Andrea; Castaño, Edilberto y Gómez, Esperanza. “La organización del estudio instrumental: un factor incidente en las molestias músculo esqueléticas en clarinetistas". Cuadernos de Música, Artes Visuales y Artes Escénicas, 11(1), 55-65, 2016. http://dx.doi.org/10.11144/Javeriana.mavae11-1.oeif 
